\title{
Fundamentos para la mejora genética de ovinos en Costa Rica
}

\author{
Andres Zúñiga Orozcoํ, Daniela Ross Moscarella ${ }^{2}$, Ayerin Carrodeguas Gonzalez ${ }^{3}$ \\ 1.Docente e Investigador. Carrera de Ingeniería Agronómica. UNED. San Pedro, San José, Costa Rica; \\ azunigao@uned.ac.cr \\ 2.Estudiante. Carrera de Ingeniería Agronómica. UNED. San Pedro, San José, Costa Rica; \\ danymoscarella@gmail.com \\ 3.Investigadora. Instituto de Investigaciones Hortícolas Liliana Dimitrova. Mayabeque. \\ Cuba,genetica2@lilliana.co.cu \\ Recibido: 04 de Noviembre de 2020 Aceptado: 30 de Junio de 2021
}

\begin{abstract}
Sheep production is an activity of growing importance in Costa Rica, as such, it requires a lot of information transfer and local research development. Local information relevant to developing new breeds with better attributes and genetic gain is scarce. This review addresses the different genetic bases to facilitate the establishment of a sheep genetic improvement program. Various options are described to carry out the selection process and a proposal is made of different crosses in sheep to obtain genetically superior animals. The aforementioned crosses are proposed to be carried out with sheep species already established in the country, however, it is applicable in other latitudes according to the improvement objectives.
\end{abstract}

Key words: crossover, sheep, selection, genotype, artificial insemination

\section{RESUMEN}

La producción de ovinos es una actividad de importancia creciente en Costa Rica, como tal, necesita de mucha transferencia de información y desarrollo de investigación local. La información local pertinente para desarrollar nuevas razas con mejores atributos y ganancia genética es escasa. En la presente revisión se abordan las diferentes bases genéticas para facilitar el establecimiento de un programa de mejoramiento genético ovino. Se describe varias opciones para realizar el proceso de selección y se hace una propuesta de diferentes cruces en ovinos con el fin de obtener animales genéticamente superiores. Los cruces mencionados, se proponen efectuar con especies ovinas ya establecidas en el país, sin embargo, es aplicable en otras latitudes según los objetivos de mejora.

Palabras claves: cruzamiento, ovinos, selección, genotipo, inseminación artificial 


\section{INTRODUCCION}

Aunque la carne de ovinos no es una opción cultural prioritaria en Costa Rica, el aumento de la demanda y sus subproductos ha tenido un creciente incremento durante los últimos años. Esta actividad se considera de importancia puesto que viene a dar un dinamismo económico en zonas rurales, creación de nuevos empleos y mejores oportunidades económicas para los productores (Mora-Valverde y Chacón-Villalobos, 2015). Además, abren un potencial para el crecimiento culinario de diversos países. También el crecimiento de esta actividad se le atribuye a la implementación de diversas actividades económicas como el turismo, las grandes cadenas de supermercados, hoteles y restaurantes, los cuales solicitan este tipo de carne para satisfacer la demanda de turistas extranjeros.

En Costa Rica, desde 1964 a la fecha, se ha reportado un aumento en la producción y consumo de productos ovinos (Mora-Valverde y Chacón-Villalobos, 2015). Según el VI Censo Nacional Agropecuario se reporta un total de 35800 ovejas en 1792 fincas (Censo Agropecuario, 2014). Los ovinos son animales herbívoros, rústicos y de fácil adaptación, no son exigentes en cuanto a pasturas y forrajes, consumen plantas anuales y perennes, por tanto, se pueden manejar de formas extensivas, intensivas o mixtas (Martinez et al., 2018). La producción de carne ovina en el trópico es considerada ventajosa sobre otros animales de granja dada las condiciones de pequeño rumiante y elevada fecundidad. La carne magra del ovino tiene similar contenido en grasa que el vacuno y porcino y con buena aceptación por la población (Sánchez, 1997).

El aumento de la población ovina para suplir la demanda ha traído consigo múltiples retos que se deben enfrentar con mejoras en el paquete tecnológico y buenas prácticas agropecuarias. De acuerdo con lo anterior, es tan importante la genética del animal como el manejo general de una finca para permitir expresar al máximo la productividad de los animales. Además, es de especial interés contar con animales de alto valor genético, para lo cual es necesario utilizar desde técnicas convencionales hasta técnicas biotecnológicas. Una herramienta importante en la producción ovina es el mejoramiento genético. Tradicionalmente se ha efectuado por medio de cruces y selección de individuos para obtener nuevas progenies con mejores características, tanto fisiológicas como genéticas, con el objetivo de alcanzar una mejor adaptación a diferentes zonas. Actualmente se cuenta con múltiples técnicas biotecnológicas para apoyar los proyectos de mejoramiento en ovinos tales como: inseminación artificial, fertilización in vitro y transferencia de embriones (Bravo, 2012).

Contar con objetivos claros desde el inicio facilitan mucho la implementación de un programa de mejora genética, así como una buena caracterización de razas, registro de información y uso de tecnología vienen a complementar la eficiencia de un proyecto de esta naturaleza. Lo anterior es importante porque permite la selección de los mejores animales dentro de un hato productivo para generar avance genético en la producción de carne ovina generación tras generación.

En la presente revisión se hace una recopilación del estado del arte sobre la actividad de mejora genética en Costa Rica, describiendo los cruces más utilizados y haciendo una propuesta para que los productores puedan enrumbar un programa, siempre recomendando un asesoramiento profesional. En primer lugar, se describen las características generales de las razas más comunes para ser usadas como pool genético, en segunda instancia de describen conceptos claves para incursionar en un proyecto de mejora genética. Posteriormente se hace una descripción de cruzamientos y técnicas de selección utilizadas comúnmente. Seguidamente se describe la importancia de la biotecnología con algunos prácticos ejemplos en los que se aplica para la mejora genética animal, y finalmente se hace una propuesta de cruzamiento para ser considerada e implementada en finca. 


\section{Material genético: un abanico de opciones múltiples}

Las razas de ovinos para carne usualmente se producen en sistemas de semiestabulado o estabulado. Estas razas se pueden dividir en dos sub grupos: razas ovinas de carne mejoradas y razas ovinas de carne rústicas, ésta última se refiere al uso de razas de línea pura. En Costa Rica las razas más utilizadas para producción y mejoramiento se muestran en la Figura 1.
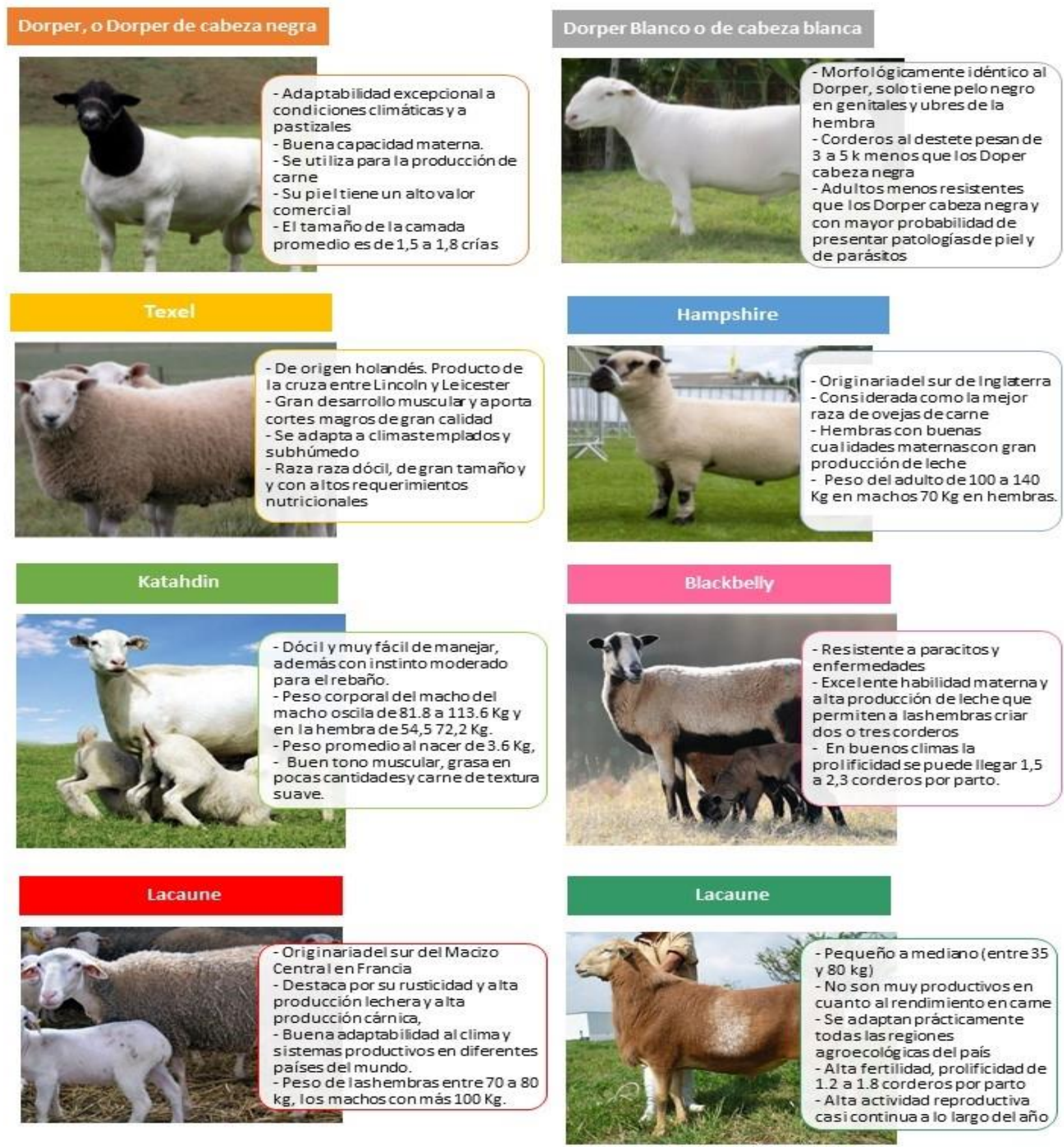

Figura 1. Razas de ovinos utilizadas en Costa Rica para producción y mejoramiento. Fuente: Centro 
Agropecuario el Remanso, 2019; APCO, 2019; Gonzales et al., 2017; Chacón, 2016; Granja Ovina San Jacinto, 2019; De la Barra et al., 2019; Galina et al., 1996.

\section{Conceptos básicos y el proceso de mejora}

El mejoramiento genético es un proceso que se puede aplicar a cualquier especie o población y se basa en el cruzamiento y selección de los mejores individuos según caracteres de importancia económica. El objetivo se basa en cambiar la frecuencia de los alelos que comúnmente son encontrados en los genes y sustituir con alelos más favorables o que en su conjunto puedan producir más que las razas puras.

La primera etapa de un programa de mejora genética en animales consiste en la definición de los rasgos a seleccionar, los que deben tener algún valor productivo, ser heredables y de fácil medición bajo procedimientos estandarizados (Cunnigham, 1974; Quintanilla-Medina et al. 2018; De la Barra et al., 2019).

En el mejoramiento genético clásico, la selección de los mejores individuos se realiza por medio de características visibles o medibles en el animal. Ese conjunto de caracteres incluyen aspectos como: longevidad, fertilidad, prolificidad, peso, crecimiento y la calidad de la carne, este conjunto de características deseables es lo que se conoce como fenotipo. El fenotipo de un organismo está dado por su genotipo, por el ambiente y por la interacción entre el genotipo y el ambiente. El genotipo no es más que la información genética de un organismo, que está contenida en el ADN. En cuanto al ambiente, abarca todos los factores a los que este expuesto el animal, como las condiciones presentes en el año y periodo de parto o nacimiento del animal, las condiciones de la finca o predio, la edad y número de partos alcanzados, alimentación y nutrición, edad en los tiempos de pesaje, condiciones sociales y salud del hato.

En la segunda etapa del proceso se elige la raza a mejorar. Usualmente esta elección se realiza antes de comenzar el programa de selección, aunque es necesario reevaluar si generará mayores beneficios el sustituir la raza en cuestión por otra con mayores atributos para ese ambiente (Piñeira et al., 2009).

La tercera etapa consiste en estudiar el grado de heredabilidad de los caracteres propuestos para el programa de mejora. Si no son heredables, dicho programa será poco eficiente y deberán emplearse técnicas alternativas a la selección (Cunnigham, 1974) La heredabilidad es la proporción de la variación de caracteres biológicos en una población atribuible a la variación genotípica entre individuos. Ofrece indicaciones del grado en que un carácter fenotípico está determinado genéticamente y puede ser exitoso o no en heredarlo (Universidad de Navarra, 2019). Si la heredabilidad tiene un valor medio o alto, se debe continuar con el programa de mejoramiento a través de un proceso de selección o de cruzamiento y posterior selección, de lo contrario deberán emplearse técnicas alternativas a la selección (Cunnigham, 1974).

La cuarta etapa consiste en definir la estrategia que se va a emplear según el valor de la heredabilidad del rasgo en el que se basa el programa de mejora. Si la heredabilidad es alta, un programa basado en la selección de ese carácter sería lo más sencillo y eficaz, pero si la heredabilidad es media o baja, es necesario evaluar la existencia de vigor híbrido (heterosis) en el cruzamiento y determinar si un sistema de cruzamiento sería apropiado. La heterosis o vigor híbrido se refiere a la posibilidad de obtener mejores individuos por la combinación de virtudes de sus padres. Entre menos emparentados son sus padres mejor resultado se obtiene. Describe la mayor fortaleza de diferentes características en la progenie o híbridos (Ravagnolo, 2005; Piñeira et al., 2009).

La quinta etapa consiste en optimizar el programa de selección, maximizando el progreso genético por unidad de tiempo hacia el objetivo propuesto, para lo cual se podría considerar la aplicación de técnicas reproductivas como inseminación artificial, fertilización in vitro y/o transferencia embrionaria (Piñeira et al., 2009). 
El principal problema que existe para identificar aquellos individuos genéticamente superiores es que el valor genético no se puede observar a simple vista. Lo que nosotros observamos en un animal es lo que se conoce como fenotipo y el mismo, está dado tanto por la constitución genética del animal como por el medio ambiente en el cual se desarrolla. Si un animal se desenvuelve de forma óptima en un medio ambiente favorable, este podrá expresar todo su potencial genético; sin embargo, si el medio ambiente en el que le toca desarrollarse no es favorable, lo más probable es que su desempeño se califique como indeseable, aunque tenga un buen potencial genético. De esta forma, para poder evaluar genéticamente a un animal, necesitamos ser capaces de poder determinar qué proporción de su comportamiento productivo se debe a su constitución genética y qué proporción se debe al medio ambiente en el cual se desarrolló.

El progreso genético que se pueda lograr al seleccionar individuos identificados como genéticamente superiores (Figura 2), va a depender en mucho de la precisión con que podamos separar la parte genética de la parte ambiental al evaluar el animal, lo cual no es una tarea fácil ni económica.

Para que el pequeño productor pueda lidiar con esta limitante se recomienda utilizar machos previamente probados, puesto que el productor pequeño e incluso mediado, carece de las herramientas, espacio y recursos para hacer estas evaluaciones. Por otra parte, es posible utilizar metodologías elaboradas para la evaluación del potencial de machos/hembras en cada explotación pecuaria (Canto, Muñoz y Reyes, 2012; Rodríguez, 2012; Aké et al., 2017).

Según Mayorga, Levy y González (2010) y Aké et al. (2017) los pasos que se deben tomar en consideración para incursionar correctamente en un programa de mejora son:

1. Definir el objetivo de selección según el interés económico, considerando si se desea realizar un sistema productivo, de crecimiento, o rendimiento en canal.

2. Definir las características que nos llevan a alcanzar el objetivo. Algunos caracteres de interés son: prolificidad, supervivencia, peso corporal, ganancia de peso o ganancia de canal.

3. Identificar genéticamente los mejores animales para esas características, las cuales requieren información previa, toma de datos pruebas de comportamiento y evaluación genética.

4. Diseminar la genética de los animales identificados como superiores.

5. Evaluar el proceso genético. 


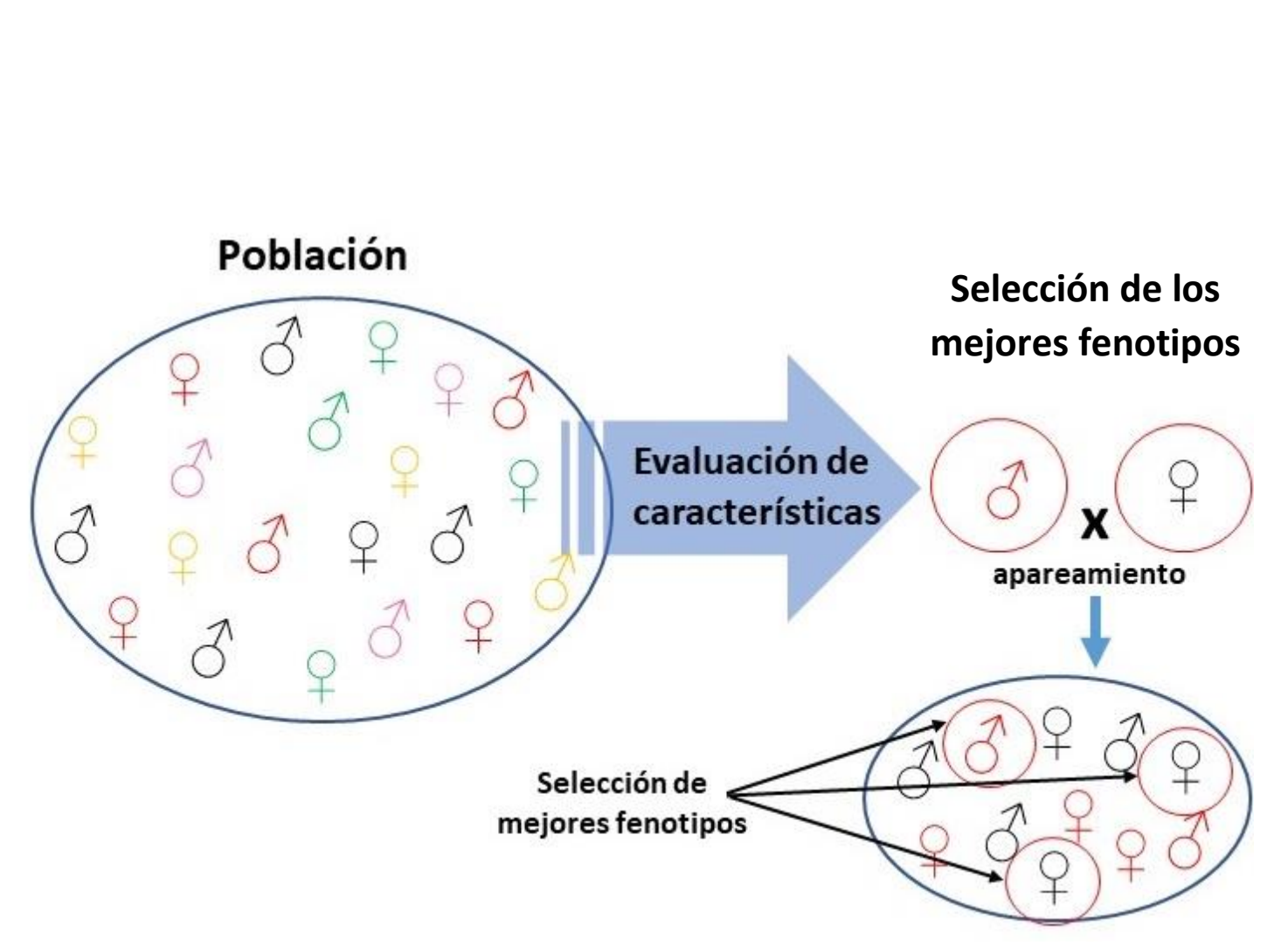

Figura 2. Esquema general de un programa de mejora genética en ovinos. Fuente: Propia

\section{Tipos de cruces en ovinos}

Los cruces son una herramienta que permite tener resultados a corto plazo y una respuesta rápida, ya sea en la primera generación (F1) o en programas que incluyan cruces terminales/comerciales según la cantidad de generaciones deseadas. La elección de cuál cruce se debe realizar, varía según las necesidades del productor y de los alcances que se quieren lograr. Es muy importante tener en cuenta los objetivos del programa de cruzamiento y elegir el método adecuado en cuanto a estos, pues una mala elección podría generar efectos desastrosos en un rebaño (Bianchi et al., 2001; Ganzábal et al., 2002).

La diferencia entre los cruces comerciales y de mejoramiento genético corresponde a que, los cruces de mejoramiento buscan reproductores con características genéticas mejoradas para obtener una línea superior en características específicas; mientras que los cruces comerciales o sistemáticos se caracterizan por obtener un producto final o comercial, no para reproducción, y se espera que estos animales tengan características que le permitan un crecimiento rápido y llegar a un peso adecuado al final del periodo de engorde. Además, se busca que tengan buenas condiciones de sanidad para que estén listos para colocarlos en el mercado.

A continuación, se exponen las 2 categorías principales de cruce y de ahí derivarán otra serie de rutas:

1. Cruza de mejoramiento: buscan generar variabilidad para encontrar una progenie que mejore el hato actual, algunos individuos son seleccionados como parentales para la siguiente generación y muchos son descartados. Aquí es recomendable aprovechar la complementariedad entre razas y explotar el vigor hibrido o heterosis. Los cruzamientos para mejoramiento se pueden dividir en dos: cruces absorbentes para tener una raza exótica dentro del predio o se puede hacer cruces criss-cross o alternados para ir formando la raza con las características deseadas. 
1.1 Cruzamiento alternado: el sistema busca llegar a obtener un producto comercial mediante el cruzamiento alternado de razas, siempre buscando que se conserven los mejores caracteres de las razas (Fig.3A). Se puede usar para mejoramiento y comercial, en el primer caso se alterna hasta que los animales seleccionados tengan las características deseadas, mientras en el segundo caso se vende lo que no se seleccione siempre y cuando cumpla con parámetros del mercado.

1.2 Cruzamiento absorbente: el sistema busca suplantar o eliminar una raza que no es deseada o que no está aportando lo suficiente, para ello se utiliza alguna raza exótica que si cumpla con los requerimientos del mercado (Fig.3B). Puede hacerse por inseminación o teniendo un semental de alto valor genético. Este método requiere persistir en el cruzamiento por varios años, hasta que la raza original prácticamente desaparece y da paso a un rebaño de la raza con que se hizo la absorción (de la Barra et al., 2012). Es usado en mejoramiento para tener disponible la raza de cruza, pero los animales resultantes de la segregación se pueden comercializar.

\begin{tabular}{|c|c|c|c|c|c|c|c|c|}
\hline $\mathbf{A}$ & A & B & & B & A & B & & \\
\hline F1 & $50 \%$ & $50 \%$ & X B & F1 & $50 \%$ & $50 \%$ & & B \\
\hline F2 & $25 \%$ & $75 \%$ & $\times A$ & F2 & $25 \%$ & $75 \%$ & $x$ & B \\
\hline F3 & $62.5 \%$ & $37.5 \%$ & X B & F3 & $12.5 \%$ & $87.5 \%$ & $x$ & B \\
\hline F4 & $31.3 \%$ & $68.7 \%$ & $\times A$ & F4 & $6.3 \%$ & $93.7 \%$ & $x$ & B \\
\hline F5 & $65.8 \%$ & $34.2 \%$ & X B & F5 & $3.3 \%$ & $96.7 \%$ & & B \\
\hline
\end{tabular}

Figura 3. A: Cruzamiento industrial terminal o comercial, ejemplificado con una técnica cíclica o alternada criss-cross. Siempre se utilizan líneas puras de forma alternada por parte de la vía paterna, mientras que las hembras se seleccionan de la progenie, lo cual permite retener un porcentaje de heterosis individual y materna. B: Diagrama cruzamiento absorbente o sustitución. Fuente: Propia

2. Cruzamiento industrial o comercial: el sistema busca principalmente enrumbar la variabilidad obtenida en el cruce hacia un animal que pueda obtener marcadamente ganancia genética y ser vendido de forma rápida. En este tipo de cruces se pueden seguir varios esquemas, como el cruzamiento alternado y cruzamientos con varias razas con un objetivo final comercial.

2.1 Cruzamiento terminal: este método tiene como principal objetivo modificar rápidamente las características productivas del rebaño, pero sin llegar a introducir los genes de la raza del carnero en el rebaño (Fig. 4), es un tipo de cruzamiento comercial. Para esto se cruzan ovejas de la raza base del plantel con carneros de una raza distinta poseedora de atributos deseables (Bianchi et al., 1998). Las crías generadas por este tipo de cruzamiento se deben destinar a la venta, y no se deben ser utilizadas como reposición o reproductores (Bravo y Romero, 2012). Es un cruce exclusivo para 
la comercialización. En la figura 4 se ilustra un ejemplo de cruce terminal con 3 razas y en la figura 5 se realiza un ejemplo con nombres de raza probados en Costa Rica.

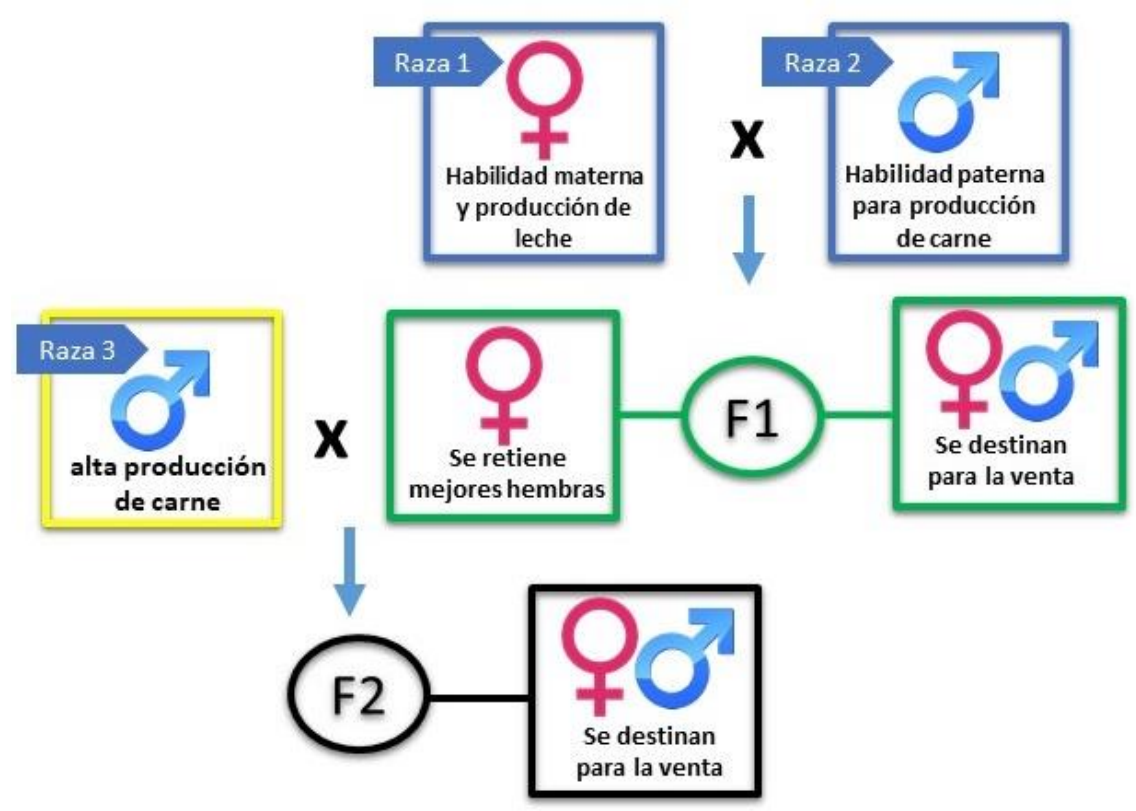

Figura 4. Diagrama de cruce Comercial terminal con 3 razas. Fuente: Propia

\section{Selección de individuos: un resultado favorable en la producción de nuevas progenies}

El proceso de selección permite determinar cuáles son los animales que cuentan con las características adecuadas para convertirse en los nuevos progenitores de una nueva generación, este proceso debe realizarse en la progenie resultante de un cruce entre razas, creando así nuevas variaciones dentro de la población (Bravo y Romero, 2012).

La selección permite tomar los mejores representantes del hato, cruzarlos y mantener la pureza de la raza o la línea pura, así como también generar nuevas razas o líneas superiores. Para garantizar una mejor decisión de la selección se deben llevar registros de los animales que aporte datos fiables, el cual debe ser analizado por un experto para identificar los factores que pueden ser mejorados dentro del hato; ésta práctica dentro de una producción extensiva permite mejorar visiblemente los rendimientos. También se debe tener un objetivo claro, el cual puede ser diferente en distintos programas, pues este depende de la estrategia comercial definida (De la Barra y Uribe, 2009) El objetivo debe ser medible y visible desde un punto de vista económico; es decir, la mejora sólo tiene sentido si es realizada para aumentar el retorno económico del plantel ganadero (Carvajal et al., 2011).

Los resultados de la sección no se esperan observar en el corto plazo, se pueden apreciar en periodos de mediano y largo plazo por lo cual la toma de registros, el análisis de la información y la buena toma de decisiones es crucial para no retroceder en el mejoramiento, así como no extender de más las generaciones necesarias para mejorar el hato.

La selección artificial es el sistema más eficiente para mejorar el comportamiento productivo de un individuo mediante el estudio de los caracteres de su descendencia. Es por ello, que la prueba de progenie 
se puede llevar a cabo sólo cuando el animal escogido haya alcanzado la madurez sexual y tenga descendencia. Generalmente la prueba se hace en los machos, pero también puede realizarse en las hembras, especialmente en la actualidad por transferencia de embriones se pueden tener muchas crías de una sola madre y así saber cuál es su valor genético. Los descendientes de diferentes animales se evalúan para determinar cuál grupo es superior y escoger así el progenitor de mejor calidad como reproductor.

Una de las herramientas más importantes en la selección de animales es el índice de selección, por cuanto tiene la particularidad de construir un criterio por múltiples variables, de preferencia con una correlación de alta magnitud positiva. De acuerdo con lo anterior, se considera información del registro fenotípico del animal y de sus parentales. Una desventaja de este método es que, si la heredabilidad de los caracteres es baja, entonces la ganancia genética que se podría conseguir puede ser poco apreciable, es por lo anterior, que se debe revisar literatura previamente, revisar registros del predio o hacer evaluaciones de heredabilidad in-situ para procurar tener un avance real (Lembeye et al., 2014).

La selección puede ser realizada por varios métodos, a continuación de describen de manera general:

Selección Fenotípica: es la que se hace cuando se compara entre sí el comportamiento fenotípico de cada uno de los miembros de un grupo de animales. Se ejecuta de forma rápida y sencilla, ciertas características no son visibles por lo que se debe recurrir a registros. La desventaja de este tipo de selección es que, comúnmente se seleccionan animales que en apariencia son buenos, pero no necesariamente; estos animales pueden verse bien por un buen manejo solamente y cuando dan descendencia no se obtiene lo deseado. Algunos criadores mantienen hasta hoy esta forma de enfrentar el mejoramiento genético (Chacón, 2016; Cruz, 2018).

Selección por información de parentales o pedigrí: en este método se seleccionan animales jóvenes, de los cuales se conocen los méritos de los progenitores y abuelos y el grado de heredabilidad de las características seleccionadas. Mientras más altos sean los grados de heredabilidad de las características seleccionadas, mucho más valor tendrá la selección por pedigrí. Se deben consideras características no presentes en el individuo al momento de seleccionar, como la cantidad de leche por hembra, o no reconocibles en vivo, como la calidad en canal (Lembeye et al., 2014; Cruz, 2018).

Selección por prueba de progenie: Este sistema de selección es el más preciso para determinar el comportamiento productivo de un individuo mediante el estudio de los caracteres de su descendencia. Es por ello, que la prueba de progenie se puede llevarse a cabo sólo cuando el animal escogido haya alcanzado la madurez sexual y tenga descendencia (Cruz, 2018).

Selección por evaluación genética: se aplica cuando se compara un grupo de animales, no por su comportamiento, sino por el de su progenie o descendencia (Cruz, 2018). Este tipo de selección debe contar con un estudio previo de registros del espécimen y sus parentales. Con los registros se permite predecir el genotipo de un individuo a partir del fenotipo de numerosos descendientes y ascendientes (Lembeye, et al., 2014). Con los registros se puede emplear modelos matemáticos que corrigen factores ambientales o contorno, que puedan variar el desempeño del animal, este sistema de selección es él que llega a tomar más tiempo para completarse, pero es el que brinda más seguridad.

Selección asistida por marcadores moleculares: este método se basa en encontrar marcadores moleculares asociados con la presencia de una característica productiva. Los marcadores moleculares son sitios que están distribuidos a lo largo del ADN, por tanto, permiten identificar en edades tempranas los individuos con rasgos deseables. Los marcadores moleculares se han convertido en una herramienta poderosa en la selección animal y permite maximizar el progreso genético para las características de interés productivo y económico en un tiempo reducido (Guimaráes et al., 2007; López - Zavala, 2010). 


\section{Biotecnología al alcance de la mejora genética}

La producción pecuaria es importante para el crecimiento económico de los países en desarrollo y la aplicación de la biotecnología está dada en gran medida por consideraciones comerciales y objetivos socioeconómicos, con el fin de aumentar productividad (Madan, 2005).

La biotecnología de la reproducción comprende técnicas como: inseminación artificial y preservación de semen, usadas para el mejoramiento genético del ganado; sexado de espermatozoides, superovulación, transferencia de embriones, producción de embriones in vitro y clonación, usadas para mejorar la eficiencia reproductiva y la tasa de reproducción (Madan, 2005).

Es preciso mencionar que las tecnologías utilizadas para el mejoramiento genético juegan un papel de incalculable trascendencia por tener la posibilidad de reducir el número de generaciones, reducir costos a largo plazo y mejorar la toma de decisiones en los cruces. A continuación, se mencionan los métodos más usados:

Inseminación artificial: La inseminación artificial, permite incrementar notablemente el aprovechamiento de un reproductor, al poder obtener un gran número de crías del mismo padre. Esto es posible debido a que, mediante un adecuado fraccionamiento del semen colectado, es posible obtener un número de crías identificadas en su paternidad (Gibbons et al., 2008).

Fertilización in vitro (FIV): Es la unión del óvulo con el espermatozoide en el laboratorio de forma in vitro, con el fin de obtener embriones ya fecundados para transferir al útero materno y que evolucionen hasta conseguir un embarazo o preñez en el animal (Ravagnolo, 2005; Gibbons et al., 2008).

Transferencia embrionaria (TE): Es la introducción de los embriones en el útero materno. Justo antes de la Transferencia Embrionaria se colocan los embriones seleccionados en medio de cultivo específico. Los embriones se cargan en el extremo del catéter de transferencia y se depositan suavemente dentro de la cavidad uterina (Ravagnolo, 2005; Martinez-Rojero, et al., 2017). Junto a la fertilización in vitro, esta técnica conviene usarla para la evaluación de la habilidad genética de animales o incrementar cantidad de animales por unidad de tiempo.

\section{Biología molecular aplicada al mejoramiento genético de ovinos}

Los programas de mejoramiento genético en ovinos usualmente consisten en la selección de individuos con las mejores cualidades genéticas, basándose en su fenotipo y genealogía, para después realizar apareamientos entre individuos con las mejores cualidades reproductivas (Ocampo et al., 2017). Sin embrago, para aumentar la competitividad del sector es necesario la incorporación de nuevas tecnologías (Ospina y Grajales, 2011), tal es el caso del uso de marcadores moleculares para la selección asistida. Estas herramientas apoyan la selección de individuos con rasgos deseables a través de la identificación, mapeo y análisis del polimorfismo de los genes implicados en las principales vías metabólicas relacionadas con el crecimiento animal y la distribución de los nutrientes en los tejidos (Coria et al., 2018).

La calidad de la carne es uno de los rasgos más perseguidos en los programas de mejora en ovinos, se ha estudiado previamente que está estrechamente relacionada con los genes de la calpaína (CAPN) y la calpastatina (CAST) (Georgieva et al., 2015). El sistema calpaína en el músculo esquelético comprende dos proteasas calcio dependientes, la calpaína 1 y 2 , y un tercer polipéptido, la calpastatina, cuya única función 
conocida es inhibir las dos calpaínas. Las calpainas también están relacionadas con el crecimiento muscular en diferentes etapas de desarrollo y con la degradación de las proteínas musculares, produciendo la pérdida de masa muscular (Bagatoli et al, 2013). Se han identificado SNPs (single nucleotide polymorphism o polimorfismo de un solo nucleótido) asociados a estos genes, los que los convierte en marcadores moleculares muy prometedores y versátiles para la selección asistida en ovinos.

Por otra parte, el gen CAPN está relacionado con el ablandamiento de la carne producto de la degradación de las proteínas miofibrilares post-morten. En otro caso, el gen CAST es el único inhibidor que se conoce de la calpaína, y su efecto es inverso, de manera que ralentiza la velocidad del ablandamiento post-morten de la carne (Knight et al., 2012).

En el año 2000, en Bovinos (Bos taurus), fue mapeado el gen CAPN1 y se encontró un QTL relacionado con la terneza de la carne. Se encontraron dos SNP ligados a este QTL (CAPN1 316 y CAPN1 530), sin embargo, estos marcadores no pudieron validarse para su uso en otras especies animales por lo que conviene realizar estudios de homología de secuencia y transcriptomica. De igual forma, se han encontrado otros SNPs, en otras especies de bovinos, relacionados a la terneza de la carne, pero hay un faltante de información e investigación para ser aplicados en selección en otras especies animales (Page et al., 2002). En ovinos, se ha estudiado un SNP relacionado al gen CAPN (g.24962426T), el cual puede ser utilizado como marcador para la selección asistida (Knight et al., 2012).

\section{Propuesta para una estrategia de cruzamiento en ovinos}

Entender los sistemas de cruzamiento permiten tomar decisiones para obtener el máximo aprovechamiento. Los autores consideran que la inducción de variabilidad inicial es un proceso de gran importancia, por lo cual la escogencia de razas es el primer paso y uno de los más cruciales, por tanto, no es para menos usar de preferencia razas puras, con alta compatibilidad y de un alto valor genético, tanto para los machos como para las hembras.

La inducción de variabilidad es una herramienta que asegura disminuir un problema con el que lidian los productores comúnmente y es la presencia de endogamia. La endogamia se presenta cuando animales altamente emparentados comienzan a reproducirse, en este caso los alelos de los caracteres pueden tener alta probabilidad de repetirse, dando como condición la homocigosis y esto puede generar defectos en los animales (De la Barra y Carvajal, 2020).

Aunque no es el enfoque de esta revisión, hay que mencionar que, en ocasiones, la endogamia puede ser beneficiosa para fijar algún carácter de alto valor. Guitou (2010) menciona que, un animal se considera $25 \%$ consanguíneo si es el resultado de un apareamiento entre hermanos completos o padres e hijos. En hermanos medios la consanguinidad es del 12,5\% debido a que existe una variación genética adicional ya que hay un solo padre / madre en común. Para evitar este efecto, cada ovino debe poseer su información de ascendencia, así se controla los cruzamientos y se selecciona los animales menos emparentados.

Por otra parte, para el caso de cruces recomendados para la industria, los autores consideran que el sistema de cruzamiento comercial basado en un cruce terminal con 3 razas es adecuado para el caso de ovinos por: facilidad en logística, relativo poco costo de inversión, compatibilidad entre las razas y permitir doble efecto de vigor híbrido. El cruce se explica en la figura 5, consiste en aparear a una hembra con características prolíficas y de buena calidad de leche como, por ejemplo, la raza Lacaune cruzada con un macho de la raza Dorper, el cual le brinda al cordero la resistencia y rápido crecimiento. En la F1 se seleccionan las mejores hembras producto de dicho cruce y se cruzan nuevamente con un semental $100 \%$ puro de la raza Hampshire por su habilidad cárnica y grosor de aplomos. En ambos cruces es recomendable la inseminación artificial 
para la facilidad de partos, uniformidad de progenie y aumento de la cantidad de individuos en la camada próxima. Si se decide utilizar un macho para monta natural debe uniformarse el celo de las hembras por preñar.

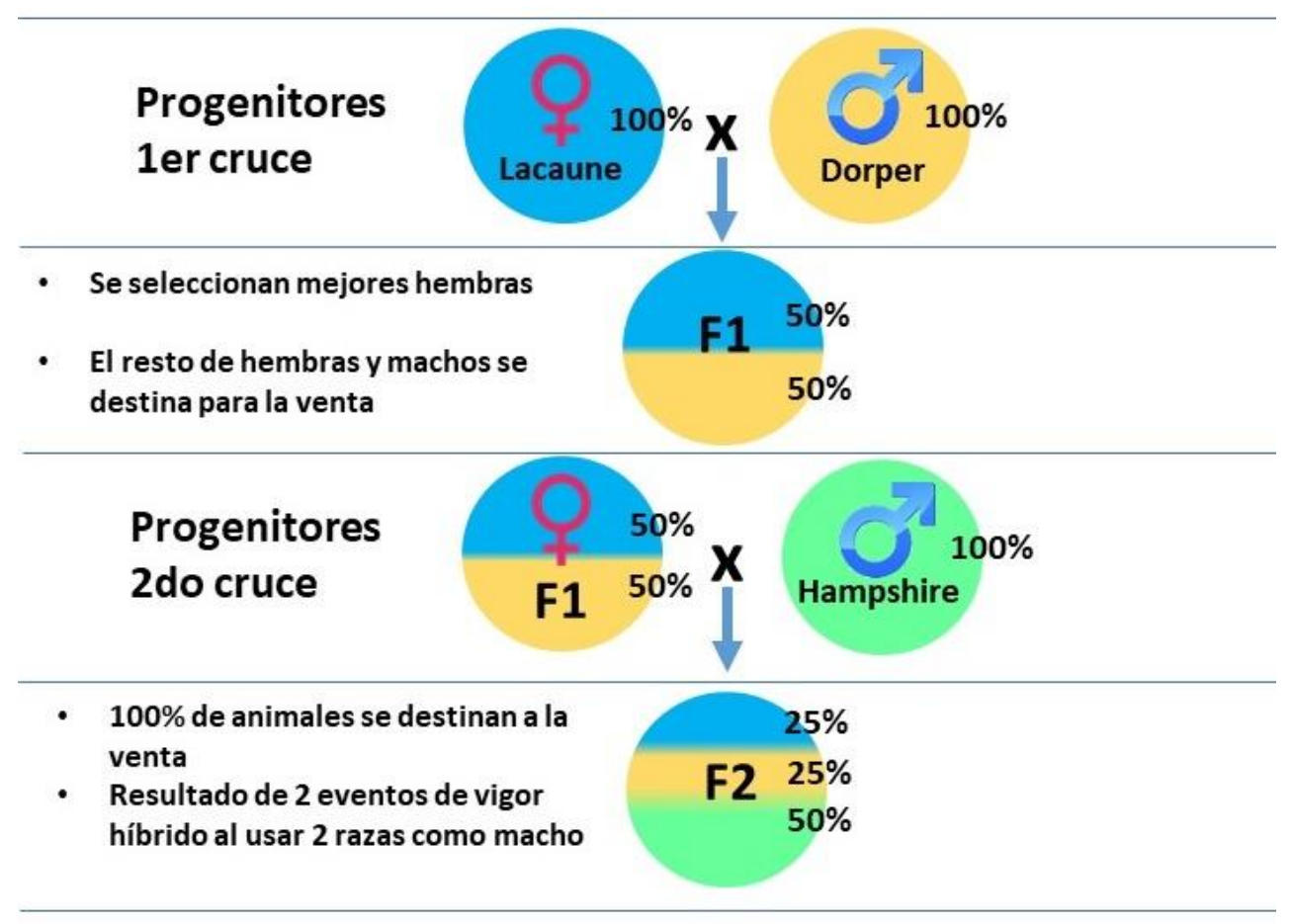

Figura 5. Diagrama de triple cruce. Fuente: Propia

Tanto el macho Dorper como el Hampshire con el que se utilizará la inseminación, deben ser ejemplares de alto valor genético, de preferencia con registro y DEPs (Diferencia esperada de la progenie) de moderado a alto mérito genético para la mayoría de caracteres de interés, según se recomienda: rendimiento de canal, peso de canal caliente, longitud de canal, circunferencia de aplomos, largo de patas, espesor de grasa dorsal, área del ojo de la chuleta, infiltración de grasa, ganancia diaria de peso, terneza de carne, peso al nacer, peso al destete, peso final, altura, conformación de paletas y pezuñas, conformación de paletas y lomo, circunferencia escrotal y una característica muy importante que es la prolificidad, es decir si proviene de madres con partos de gemelos, puesto que, en ovinos es posible mejorar este carácter para hacer líneas prolíficas (Magaña-Monforte et al., 2013; Quintanilla-Medina et al., 2018).

En este cruce, en la F2 se obtienen animales provenientes de 2 cruzamientos anteriores (Laucane x Dorper y LauDorper x Hamphsire), tanto hembras como machos estarán listos para ser llevados al matadero en 3 o 4 meses. Cabe destacar que también se puede utilizar la raza Texel en lugar de la Hampshire, ya que es compatible con la progenie F1 proveniente de cruces entre Laucane x Dorper. A continuación, en la figura 5 se presenta el esquema de mejoramiento:

Este cruzamiento permite una utilización total de la heterosis materna ya que la madre es $100 \%$ heterocigota, además de la complementariedad entre 3 razas. Permite también una utilización total de la heterosis individual ya que los descendientes son también $100 \%$ heterocigotos en ambos cruces, teniendo alelos provenientes de las 3 razas. 
Lo descrito anteriormente concuerda con otros autores como Gonzabal et al. (2007), quienes mencionan en su experimento que las canales pertenecientes a corderos triple cruza también fueron más pesadas que las de F1 y en forma similar con mayor proporción de pierna que estas últimas.

\section{Otros tipos de cruce}

Pelibuey x Dorper: en este cruce lo que se busca es obtener un animal de alta resistencia a condiciones de alta temperatura, habilidad materna y alta prolificidad, además de aportar valor cárnico por parte del Dorper. Estos animales normalmente obtenidos de un F1 son de bajo costo de operación y prolificidad (Rocha et al., 2009). Como desventaja se obtiene que no tienen gran incremento en valor cárnico como se obtiene al usar machos de las razas Suffolk, Hampshire o Texel pero son muy adaptables a zonas secas. En este caso podría valorarse incorporar una raza cárnica adicional, pero teniendo en cuenta que es muy posible tener que retrocruzar con Dorper para mejorar tolerancia a condiciones ambientales adversas.

Texel x Hampshire: este cruce es de naturaleza altamente cárnica, funciona muy bien en zonas altas, por encima de los $1800 \mathrm{msnm}$ con una estación lluviosa moderada. Si se desarrolla en zonas con alta pluviosidad puede tener problemas si no se maneja una sanidad en el esquilar el pelaje (Lara, 2015). Puede valorarse incluir una raza de tolerancia como Dorper o Pelibuey pero teniendo en cuenta que en una siguiente generación deberá incorporar nuevamente una raza cárnica en caso que se dé una disminución drástica de canal, en este caso se recomienda un cruce alternado o criss-cross hasta obtener lo deseado y no se debe olvidar tener varias líneas.

Lacaune x Dorper: en este cruce se pretende tener alta prolificidad, así como buena habilidad materna y un incremento medio en cuanto a calidad de canal. Como desventaja puede presentarse que requiera una tercera raza para introgresar tolerancia a condiciones adversas. Puede adaptarse muy bien desde los 6001800 msnm en el trópico.

Otros estudios realizados en otras latitudes en cuanto a cruzamientos describen cruces como Pelibuey $\mathrm{x}$ Blackbelly, reportando mejor peso pre y posdestete (Hinojosa-Cuéllar et al., 2013). Lara (2015) reporta cruzamientos entre Hampshire x Charollais, Hampshire x Dorset y Hampshire x Texel, obteniendo un incremento en \% de redimiento de canal y área del músculo del lomo. Gonzabal et al. (2007) reportan el cruce entre Hampshire x Suffolk obteniendo corderos un poco más tardíos, pero con mayor peso a la faena.

\section{Conclusiones:}

- El mejoramiento genético en ovinos y la demanda de la carne ovina ha tenido un crecimiento en últimos 20 años en Costa Rica. Sin embargo, hay necesidad de profesionalizar tanto la producción como las técnicas de mejora genética.

- Al desarrollar un programa de mejoramiento genético en ovinos, se debe tener en cuenta los objetivos, las características que se desean mejorar, las etapas del proceso y la duración de cada una de ellas.

- En rendimiento de cada animal va a depender de su genotipo, pero también del buen manejo para el desarrollo óptimo del potencial genético.

- Los cruzamientos propuestos abordan de una manera sencilla las opciones que los productores pueden elegir, sin embargo, no son la única opción y se puede experimentar en cuanto a nuevas combinaciones de razas y esquemas de cruzamiento. 
- Las herramientas biotecnológicas cada vez toman un papel preponderante en la efectividad de programas de mejora animal, especialmente por la reducción en tiempo y la mejora en la calidad de información que se obtiene para evaluar progenies y parentales macho/hembra.

\section{Referencias}

Aké, J.R., Aké, N.Y., Aké, J.R. y Segura, J.C (2017). Evaluación reproductiva del macho ovino. Madrid, España. Editorial Académica Española.

APCO (2019). Asociación Paraguaya de Crianza de Ovinos. Recuperado de: http://apco.com.py/?fbclid=IwAR14aqizNOhHzbSUBaIThGNrlEFoB2oIs01ELFZt8ha5dSJ2OdITsf0Zy 0

Bagatoli A, Gasparino E, Soares M, Amaral R, Macedo F, Voltolini D, et al. (2013). Expression of calpastatin and myostatin genes associated with lamb meat quality. Genet. Mol.Res., 12(4), pp. 6168- 6175.

Bianchi, G. (2001). Utilización de razas y cruzamientos para la producción de carne ovina en el Uruguay. Curso internacional en salud y producción ovina; Valdivia, Chile. 17-18 mayo 2001 Valdivia. CL. 2001. Universidad Austral de Chile. 53-69.

Bianchi, G.; Olivera, G.; Garibotto, G.; Bentancur, O.; Morros, J.; Nin, J. y Platero, M. (1998). Cruzamientos entre padres Corriedale, Texel, Hampshire Down y Southdown sobre ovejas C. Corriedale. Rev. Arg. Prod. Anim., 18(51), pp. 303-304.

Romero, O. y Bravo, S. (2012). Mejoramiento genético en ovinos. Boletín INIA - Instituto de Investigaciones Agropecuarias. Recuperado de: https://biblioteca.inia.cl/handle/123456789/7532

Canto, F., Muñoz, C. y Reyes, J. (2012). Evaluación reproductiva y sanitaria del carnero. INIA - Instituto de Investigaciones Agropecuarias. Montevideo, Uruguay. Recuperado de: https://biblioteca.inia.cl/bitstream/handle/123456789/4753/NR40695.pdf?sequence=1\&isAllowed=y

Carvajal, A., Uribe, H. y Barra, R. (2011). Objetivos de la mejora genética en bovinos de leche. Revista Agrícola, Boletín N¹04. INIA - Instituto Nacional de Investigación Agropecuaria. Montevideo, Uruguay. Recuperado

de: https://biblioteca.inia.cl/bitstream/handle/123456789/4742/NR40685.pdf?sequence=1\&isAllowed=y

Censo Agropecuario (2014). VI Censo Nacional Agropecuario. Resultados Generales. Recuperado de: http://www.mag.go.cr/bibliotecavirtual/U40-10581.pdf

Centro Agropecuario el Remanso (2019). Ganado Dorper. Papelón, Portuguesa. Venezuela. Recuperado de: https://www.centroelremanso.org/dorper/

Chacón, C. (2016). Manual Práctico de Manejo General en Ovinos para pequeños productores del municipio de Zumpango, Estado de México. Recuperado de: https://repository.ucc.edu.co/bitstream/20.500.12494/562/1/LIBRO\%20PRACTICA\%20PROFESIONA L\%20CRISTIAN\%201.pdf

Coria, M., Carranza, P. y Palma, G. (2018). Calpain system in meat tenderization: A molecular approach. Rev MVZ Córdoba, 23(1), pp. 6523-6536.

De la Barra, R. y Carvajal, A. (2020) Control de la Endogamia en rebaños de ovinos. Osorno: Informativo. INIA - Instituto de Investigaciones Agropecuarias. Santiago, Chile. Recuperado de: 
https://biblioteca.inia.cl/handle/123456789/3997

De la Barra, R. y Uribe, H. (2009). Desarrollo de núcleos genéticos ovinos. Boletín Informativo No 63. INIA Instituto de Investigaciones Agropecuarias. Santiago, Chile.

De la Barra, R., Carvajal, A., Martínez, M.E. y Palavecinos, P. (2019). Diversidad racial de la ganadería ovina en el territorio de la Patagonia verde, Chile, AICA 13, pp. 41-51

De la Barra R, Carvajal, A.M. y Uribe H. (2012). Bases para el mejoramiento genético ovino. Boletín Informativo $\mathrm{N}^{\circ}$ 244. INIA - Instituto de Investigaciones Agropecuarias.

Galina, M.A., Morales, R., Silva, E. y López, B. (1996). Reproductive performance of Pelibuey and Blackbelly sheep under tropical management. Recuperado de: https://www.sciencedirect.com/science/article/pii/0921448895008780

Gamarra, G. (2016). Genética ovina francesa. Agro Enfoque, 30(203), 34-40p.

Ganzábal, A., de Mattos, D., Montossi, F., Ranchero, G., San Julián, R., Pérez, J.A., Noboa, M., de los Campos, G. y Calistro, S. (2002). Inserción de tecnologías de cruzamientos ovinos en sistemas intensivos de producción. Boletin $\mathrm{N}^{\circ} 126$. INIA - Instituto de Investigaciones Agropecuarias.

Georgieva, S., Hristova, D., Dimitrova, I., Stancheva, N. y Bozhilova-Sakova, M. (2015). Molecular analysis of ovine calpastatin (CAST) and myostatin (MSTN) genes in Synthetic Population Bulgarian Milk sheep using PCR-RFLP. J BioSci Biotechnol, 4(1), pp. 95-99.

Gibbons, A., Pereyra, F., Silvestre, P. y Cueto, M. (2008). Vitrificación de embriones ovinos en tips. Memorias de las Primeras Jornadas Internacionales del Instituto de Investigación y Tecnología en Reproducción Animal-INITRA. Facultad de Ciencias Veterinarias. UBA. 24-26 de septiembre. Buenos Aires, Argentina.

Gonzabal, A., Montofossi, F., Ciappesoni, G., Banchero, G., Ravagnolo, O., San Julián R. y Luzardo, S. (2007). Cruzamientos para la producción de carne ovina de calidad. Resultados: comportamiento reproductivo y habilidad materna de ovejas, crecimiento y calidad de canal de corderos. INIA, Serie Técnica $\mathrm{N}^{\circ} 170$. Montevideo, Uruguay.

Gonzales K. (2019). Ganado Blackbelly. Sincelejo, Sucre, Colombia. Recuperado de: https://zoovetesmipasion.com/ovinos/razas-de-ovinos/raza-ovinos-black-belly/

Gonzales K. (2019). Ganado Hampshire. Sincelejo, Sucre, Colombia. Recuperado de: https://zoovetesmipasion.com/ovinos/razas-de-ovinos/raza-ovina-hampshire/

Gonzales, K. (2017). Razas de Ovinos Dorper. Zootecnia y Veterinaria. Sincelejo, Sucre, Colombia. Recuperado de: https://zoovetesmipasion.com/ovinos/razas-de-ovinos/raza-ovina-hampshire/

Gonzalez M., Tapia, V. y Marilyn, M. (2017) Manual de manejo ovino. Boletín INIA - Instituto de Investigaciones Agropecuarias. Recuperado de: https://biblioteca.inia.cl/handle/123456789/6668

Granja Ovina San Jacinto (2019). Granja Ovina San Jacinto, Uruguay. Recuperado de: http://granjaovinasanjacinto.com/?fbclid=IwAR30dTfK3PIgWhtqsDWOjFzCHilclTu6u2k_UlaYwwM UJFcXxNRlcvUgklg

Guimaráes, E.P., Ruane, J., Sherf, B.D., Sonnino, A. y Dargie, J. eds. (2007). Marker Assisted Selection: current status and perspectives in crops, livestock, forestry and fish. Roma: Food and Agriculture Organization of United Nations. Roma, Italia. Editorial, FAO. 
Guitou, H. (2010). Coeficiente de consanguinidad de un individuo. Sistemas de apareamiento. Rev Angus. 248, pp. 31-37.

Hinojosa-Cuéllar, J.A., Oliva-Hernández, J., Torres-Hernández, G., \& Segura-Correa, J.C. (2013). Comportamiento productivo de corderos F1 Pelibuey x Blackbelly y cruces con Dorper y Katahdin en un sistema de producción del trópico húmedo de Tabasco, México. Archivos de medicina veterinaria, 45(2), pp. 135-143. DOI: $10.4067 /$ S0301-732X2013000200004

INEC (2014). Costa Rica: Total de fincas con ganado ovino por cantidad de animales y tamaño del hato. Recuperado de: http://www.inec.go.cr/censos/censo-agropecuario-2014

Knight, M.I., Daetwyler, H.D., Hayes, B.J., Hayden, M.J., Ball, A.J., Pethick, D.W., et al. (2012). Discovery and trait association of single nucleotide polymorphisms from gene regions of influence on meat tenderness and long- chain omega-3 fatty acid content in Australian lamb. Animal Production Science, 52(7), pp. 591-600.

Lara, J. (2015). Los cruzamientos en producción ovina, experiencias prácticas en la utilización de razas cárnicas en cruces terminales. $7^{\circ}$ Congreso Internacional del Borrego. Hidalgo, México. Recuperado de: http://www.borrego.com.mx/wp-

content/uploads/2019/06/Estrategias_de_cruzamiento_para_mejorar_la_produccion_de_carne.pdf

Lembeye, F., Castellaro, G., Magofke, J.C. y Uribe, H. (2014). Comparación de criterios de selección para ovinos Suffolk Down manejados en condiciones extensivas en la zona central de Chile. Arch Med Vet 46, pp. 239-246.

López - Zavala, R. (2010). Marcadores Genéticos, su detección y utilización como herramienta de selección en el ganado bovino ¿Qué es lo que se está haciendo en Tamaulipas? CienciaUAT, 4(4), pp. 44-49.

López, A., Román, F. (2016). Catálogo De Sementales Mejorantes Lacaune 2016. Madrid, España. Revista AESLA. Asociación De Ganaderos De Ovino Lacaune. 58p.

Madan, M.L. (2005). Animal biotechnology: applications and economic implication in developing countries. Revue Scientifique et Technique Office International Des Epizooties 24(1), pp. 123-139

Magaña-Monforte, J.G., Huchin-Cab, M., Ake-López, R.J., Segura-Correa, J.C. (2013). A field study of reproductive performance and productivity of Pelibuey ewes in Southeastern Mexico. Trop Anim Health Prod 45, pp. 1771-1776. doi: 10.1007/ s11250-013-0431-2

Martínez-Rojero, R., Mejía-Villanueva, O., Zarco-Quintero, L., Mastache-Lagunas, A., \& Reyna-Santamaría, L. (2017). Evaluación de un protocolo de superovulación para transferencia de embriones en ovejas Criollas de la Montaña de Guerrero. Abanico veterinario, 7(3), pp. 30-36. https://doi.org/10.21929/abavet2017.73.3

Mayorga, F.J., Levy, J. y González, E. (2010). Guía técnica de programas de control de producción y mejoramiento genético en ovinos. Consejo Nacional de los Recursos Genéticos Pecuarios (CONARGEN), México D.F, México. Recuperado de: https://uno.org.mx/guiaovinos/guiaovinos.pdf

Mora-Valverde, D. y Chacón-Villalobos, A. (2015). La ovinocultura en Costa Rica: caracterización sectorial 2014. Nutrición Animal Tropical, 9(2), pp. 124-155.

Mujica, M. (2015). Introducción al mejoramiento genético. Recuperado de: http://aulavirtual.agro.unlp.edu.ar/pluginfile.php/39106/mod_resource/content/1/clase\%20\%202018.pdf

Ocampo, R., Martínez, R., Rocha, J., Cardona, H. (2017). Genetic characterization of Colombian indigenous sheep. Rev Colomb Ciencias Pecuarias. 30(2), pp. 116-125. 
Ospina, Ó., Grajales, H. (2011). Gestión del conocimiento: mayor producción y competitividad. Perspectivas para los sistemas de producción ovino-caprinos. Rev Med Vet, 22(2), pp. 95-113.

Page, B.T., Casas, E., Heaton, M.P., Cullen, N.G., Hyndman, D.L., Morris, C.A., et al. (2002). Evaluation of single-nucleotide polymorphisms in CAPN1 for association with meat tenderness in cattle. J. Anim. Sci. 80, pp. 3077-3085.

Piñeira, J., Riveros, J., Felmer, R. (2009). Herramientas de última generación para mejoramiento genético animal. Tierra Adentro. Recuperado de: https://biblioteca.inia.cl/handle/123456789/5060

Quintanilla-Medina, J.J., González-Reyna, A., Hernández-Meléndez, J., Limas-Martínez, A.G., Carreón-Pérez, A., \& Martínez-González, J.C. (2018). Producción de ovinos de pelo bajo condiciones de pastoreo en el noreste de México. Revista de Investigaciones Veterinarias del Perú, 29(2), pp. 544-551. https://dx.doi.org/10.15381/rivep.v29i2.13863

Ravagnolo, O., Ciappesoni, G., Aguilar, I., Pravia, M. (2005). Mejoramiento Genético Animal, herramienta para un crecimiento permanente. Revista INIA (2), pp. 6-9.

Revista del Borrego (2013) Ganado Katahdin. Mazatlán, Sinaloa, México. Recuperado de: https://docplayer.es/18156822-Katahdin-edicion-especial-de-la-revista-del-borrego-raza-suprema-deovinos-de-pelo.html

Rocha, L.P., Fraga, A.B., Aráujo, J.T., Figueira, R.F., Pacheco, K.M.G., Silva, F.I. y Rodríguez, D.S. (2009). Desempenho de cordeiros cruzados em Alagoas, Brasil. Arch. Zootec. 58(221), pp. 145-148.

Rodríguez, L. (2012). Evaluación del efecto del macho sobre la fertilidad de ovejas Merino encarneradas en otoño. Tesis de Grado. Universidad de la República, Montevideo, Uruguay.

Roldan, G. (2014). Mejoramiento animal. Recuperado de: http://agro.unc.edu.ar/ mejoramientoanimal/wpcontent/uploads/2014/10/PRACTICO-2-BASES-DEL-MEJORAMIENTO.pdf

Romero, Y., Oriella y Bravo, M.S. (2012). Mejoramiento genético en ovinos. Temuco: Boletín INIA - Instituto de Investigaciones Agropecuarias. Recuperado de: https://biblioteca.inia.cl/handle/123456789/7532

Sánchez, A. (1997). Alimentación durante la gestación. Ovinos tropicales en el Cantón Quevedo: Universidad Técnica de Quevedo. Recuperado de: https://www.redalyc.org/pdf/636/63612657010.pdf

Squella, F., Uribe, H. y Muñoz, C. (2009). Simposio de Recursos Genéticos para América Latina y el Caribe. Evaluación de razas y cruzas ovinas en el secano Mediterráneo Central de Chile. SIRGEALC, Pucón, Chile.

Universidad de Navarra (2019). Heredabilidad. Madrid, España. Recuperado de: https://www.cun.es/diccionariomedico/terminos/heredabilidad

Vásquez, N. (2011). Producción de ovinos de Carne en Costa Rica: estudio de factibilidad técnica y económica para la implementación de un modelo productivo. Tesis Lic. San José, CR. UCR. 308p.

Videla, J. (2014). Mejoramiento genético en animales domésticos. Editorial MACRO. Lima, Perú. 143p. 